\title{
Conversion of Continued Fractions into Power Series
}

\author{
By A. J. Zajta and W. Pandikow
}

Abstract. In Section 1, continued fractions of the special form

$$
1]-\frac{z_{1} t}{\Gamma 1}-\frac{z_{2} t}{\Gamma 1}-\frac{z_{3} t}{\Gamma 1}-\cdots
$$

are considered, and a general formula is given for the coefficients of the power series corresponding to (1). In Section 2, the problems of programming the computation of coefficients are discussed.

The continued fraction (1), or its variants, has been studied by many distinguished mathematicians. The problem of converting (1) into a power series has also been considered, and a number of partial results are known. A detailed account can be found in Perron [1].

In this paper, we will not make use of the techniques that are generally applied in the theory of continued fractions. Instead, our approach employs some simple combinatorial and probabilistic arguments. Nevertheless, the methods are quite elementary and can be understood even by those who are unfamiliar with probability theory.

1. The conversion formula is expressed in the following THEOREM. The coefficients $F_{n}\left(z_{1}, z_{2}, \cdots, z_{n}\right)$ of the power series

$$
1+\sum_{n=1}^{\infty} F_{n}\left(z_{1}, z_{2}, \cdots, z_{n}\right) \cdot t^{n}
$$

associated with the continued fraction (1), have the following explicit form:

$$
\sum z_{1}^{e_{1}} \prod_{k=2}^{n}\left(\begin{array}{c}
e_{k-1}+e_{k}-1 \\
e_{k}
\end{array}\right) z_{k}^{e_{k}}
$$

where the exponents $e_{k}(k=1,2, \cdots, n)$ are nonnegative integers and the summation is to be extended over all partitions of the positive integer $n$, with parts $e_{k}$,

$$
\sum_{k=1}^{n} e_{k}=n
$$

The proof is carried out in several steps. First, we introduce a finite $n$-termed sequence $\left\{\gamma_{i}\right\}_{1}^{n}$ of random variables $\gamma_{i}, i=1,2, \cdots, n$, defined by the following properties:

Received August 20, 1973.

AMS (MOS) subject classifications (1970). Primary 05A1 5, 40-04, 41-04; Secondary 05 A17.

Key words and phrases. Continued fractions, power series, conversion formula, partitions, random variables, random walk with a barrier, generating functions, convolution formula, algorithm for generating partitions, pointer addresses, memory space, memory locations. 
(i)

(ii)

$$
\begin{aligned}
\gamma_{i}-\gamma_{j} & =\text { integer } & & (i, j=1,2, \cdots, n), \\
\gamma_{i} & \geqslant \gamma_{1} & & (i=2, \cdots, n), \\
\gamma_{i} & \leqslant \gamma_{i-1}+1 & & (i=2, \cdots, n) .
\end{aligned}
$$

Such sequences will be referred to as gamma sequences. The value $c$ that is actually taken by the first element, $\gamma_{1}$, will be called the floor of the sequence. Two of these sequences, $\left\{\gamma_{i}\right\}_{1}^{n}$ and $\left\{\gamma_{i}^{\prime}\right\}_{1}^{n}$, will be called equivalent, if $\gamma_{1}-\gamma_{1}^{\prime}=\gamma_{2}-\gamma_{2}^{\prime}=\cdots=$ $\gamma_{n}-\gamma_{n}^{\prime}$. This defines an equivalence relation, under which all these gamma sequences (for each fixed $n$ ) fall into equivalence classes. Since every class can be represented by one single sequence belonging to it, we shall find it convenient to choose as each representative gamma sequence the "reduced" sequence, that is, the one with floor $c=1$.

Reduced sequences have a simple geometric interpretation by considering random walks on a line with a barrier. A particle starts at the origin and takes $n$ discrete steps of +1 and $n$ discrete steps of -1 , but never crosses the origin. We represent the walk graphically in the $(x, y)$-plane by a path joining the points $(0,0),\left(1, y_{1}\right), \cdots,\left(i, y_{i}\right)$, $\cdots,(2 n, 0)$. A point $P\left(i, y_{i}\right)$ of the path is called a point of increase if $y_{i}=y_{i-1}+1$. A reduced gamma sequence may then be defined as the sequence of $y$ coordinates of the points of increase.

For any reduced gamma sequence of $n$ elements, let $\nu_{k}$ denote the number of those elements $\gamma_{i}$ whose values are equal to $k$. Let us consider the following event:

$$
C_{n}\left(e_{1}, e_{2}, \cdots, e_{j}\right)=\bigcap_{k=1}^{j}\left\{\nu_{k}=e_{k}\right\}
$$

where $j \leqslant n, e_{k}>0$ and $e_{1}+e_{2}+\cdots+e_{j}=n$. This event may occur in various ways; we denote by $N\left[C_{n}\left(e_{1}, e_{2}, \cdots, e_{j}\right)\right]$ the total number of instances when $C_{n}\left(e_{1}, e_{2}, \cdots, e_{j}\right)$ occurs. The number $N\left[C_{n}\left(e_{1}, e_{2}, \cdots, e_{j}\right)\right]$ can be computed from a recursive formula, provided $N\left[C_{n^{\prime}}\left(e_{1}, e_{2}, \cdots, e_{j-1}\right)\right]$ is known. The event $C_{n^{\prime}}\left(e_{1}, e_{2}, \cdots, e_{j-1}\right)$ is related to a reduced gamma sequence of $n^{\prime}=n-e_{j}$ elements, for which the values of variables $\nu_{k}, k=1,2, \cdots, j-1$, remain the same as before. The original gamma sequence may be obtained from the sequence of $n^{\prime}$ elements by adjoining $e_{j}$ new elements, each having the same value $j$. The new elements cannot be inserted in an arbitrary order: by condition (iii), they can be adjoined consecutively or each can be adjoined immediately following an element of value $j-1$. This means that there are exactly

$$
\left(\begin{array}{c}
e_{j-1}+e_{j}-1 \\
e_{j}
\end{array}\right)
$$

different possibilities, thus

$$
N\left[C_{n}\left(e_{1}, e_{2}, \cdots, e_{j}\right)\right]=N\left[C_{n}\left(e_{1}, e_{2}, \cdots, e_{j-1}\right)\right] \cdot\left(\begin{array}{c}
e_{j-1}+e_{j}-1 \\
e_{j}
\end{array}\right),
$$


or, by induction,

$$
N\left[C_{n}\left(e_{1}, e_{2}, \cdots, e_{j}\right)\right]=\prod_{k=2}^{j}\left(\begin{array}{c}
e_{k-1}+e_{k}-1 \\
e_{k}
\end{array}\right)
$$

In what follows, we will assume that different possible paths of the random walk are equally probable. Then the joint generating function of variables $v_{k}, k=1,2, \cdots, n$, may be expressed as follows:

$$
G_{n}\left(z_{1}, z_{2}, \cdots, z_{n}\right)=\frac{F_{n}\left(z_{1}, z_{2}, \cdots, z_{n}\right)}{F_{n}(1,1, \cdots, 1)},
$$

where

$$
F_{n}\left(z_{1}, z_{2}, \cdots, z_{n}\right)=\sum N\left[C_{n}\left(e_{1}, e_{2}, \cdots, e_{j}\right)\right] z_{1}^{e_{1}} z_{2}^{e_{2}} \cdots z_{j}^{e_{j}}
$$

Here, $z_{k}$ denotes the generating function variable corresponding to $\nu_{k}$. The summation must be extended over all partitions of $n$ into positive integers $e_{k}$, i.e.,

$$
n=e_{1}+e_{2}+\cdots+e_{j}, \quad(j \leqslant n) .
$$

The functions $F_{n}\left(z_{1}, z_{2}, \cdots, z_{n}\right)$ can be computed in two different ways. On the one hand, by applying (6), we have

$$
F_{n}\left(z_{1}, z_{2}, \cdots, z_{n}\right)=\sum z_{1}^{e_{1}} \prod_{k=2}^{n}\left(\begin{array}{c}
e_{k-1}+e_{k}-1 \\
e_{k}
\end{array}\right) z_{k}^{e_{k}}
$$

On the other hand, they satisfy the following convolution formula:

$$
F_{n+1}\left(z_{1}, z_{2}, \cdots, z_{n+1}\right)=z_{1} \cdot \sum_{k=0}^{n} F_{k}\left(z_{1}, z_{2}, \cdots, z_{k}\right) \cdot F_{n-k}\left(z_{2}, z_{3}, \cdots, z_{n-k+1}\right),
$$

where we put $F_{0}=1$. Using this formula, the functions $F_{n}$ can be computed recursively.

Proof of the Convolution Formula (10). Consider a reduced gamma sequence of $n+1$ elements. By omitting the initial element (whose value is of course equal to 1 ), three possibilities may occur:

(i) The new sequence is also a reduced gamma sequence. This happens if $\gamma_{2}=1$ in the original sequence.

(ii) The new sequence is a gamma sequence with floor $c=2$. This happens if $\nu_{1}=1$ in the original sequence.

(iii) In the original sequence $\gamma_{2}=2$ and $\nu_{1}>1$. Then there exists an integer $k$ such that $2 \leqslant k<n, \gamma_{n-k+2}=1$ and $\gamma_{i}>1$ for $2 \leqslant i<n-k+2$. The new sequence breaks up into two distinct gamma sequences, $\left\{\gamma_{i}\right\}_{2}^{n-k+1}$ and $\left\{\gamma_{i}\right\}_{n-k+2}^{n+1}$. The first has the floor value $c=2$ and the second is a reduced sequence.

Case (i) contributes to the right-hand side of (10) by $z_{1} \cdot F_{n}\left(z_{1}, z_{2}, \cdots, z_{n}\right)$, case (ii) contributes by $z_{1} \cdot F_{n}\left(z_{2}, z_{3}, \cdots, z_{n+1}\right)$, and, finally, case (iii) contributes by 


$$
z_{1} \sum_{k=1}^{n-1} F_{k}\left(z_{1}, z_{2}, \cdots, z_{k}\right) \cdot F_{n-k}\left(z_{2}, z_{3}, \cdots, z_{n-k+1}\right) .
$$

The Generating Function for the Functions $F_{n}$. This is defined by the infinite power series:

$$
\phi_{1}(t)=1+\sum_{n=1}^{\infty} F_{n}\left(z_{1}, z_{2}, \cdots, z_{n}\right) \cdot t^{n} .
$$

Using similar notation, we introduce further generating functions $\phi_{k}(t), k=2,3, \cdots$, e.g.,

$$
\begin{aligned}
& \phi_{2}(t)=1+\sum_{n=1}^{\infty} F_{n}\left(z_{2}, z_{3}, \cdots, z_{n+1}\right) \cdot t^{n}, \\
& \phi_{3}(t)=1+\sum_{n=1}^{\infty} F_{n}\left(z_{3}, z_{4}, \cdots, z_{n+2}\right) \cdot t^{n}, \text { etc. }
\end{aligned}
$$

Now, let us form the Cauchy product of the power series of $\phi_{1}(t)$ and $\phi_{2}(t)$ and apply (10). We have

$$
\begin{aligned}
\phi_{1}(t) \cdot \phi_{2}(t) & =1+\frac{1}{z_{1}} F_{2}\left(z_{1}, z_{2}\right) \cdot t+\frac{1}{z_{1}} F_{3}\left(z_{1}, z_{2}, z_{3}\right) t^{2}+\cdots \\
& =\frac{1}{t z_{1}}\left(\phi_{1}(t)-1\right),
\end{aligned}
$$

whence $\phi_{1}(t)=1 /\left(1-z_{1} t \phi_{2}(t)\right)$, or in general,

$$
\phi_{k}(t)=\frac{1}{1-z_{k} t \phi_{k+1}(t)}, \quad(k=1,2, \cdots) .
$$

Using this formula repeatedly, we have the representation of $\phi_{1}(t)$ in a continued fraction:

$$
\phi_{1}(t)=\frac{1}{1}-\frac{z_{1} t}{\Gamma 1}-\frac{z_{2} t}{\mid 1}-\frac{z_{3} t}{\Gamma 1}-\cdots .
$$

Hence the theorem.

COROLlaRY. The total number of different possible paths of random walks which were described earlier in this paper, is equal to $(1 /(n+1))\left(\begin{array}{c}2 n \\ n\end{array}\right)$.

Proof. Clearly, the total number of paths is given by $F_{n}(1,1, \cdots, 1)$ and this can be computed as the coefficient of $t^{n}$ in the power series associated with $\phi_{1}(t)$ when $z_{k}=1$ for all $k$. But then $\phi_{k}(t)=\phi_{1}(t)$ for all $k$ and hence by (13)

$$
t \cdot \phi_{1}^{2}(t)-\phi_{1}(t)+1=0 .
$$

Thus

$$
\begin{aligned}
\phi_{1}(t) & =\frac{1-\sqrt{1-4 t}}{2 t}=\frac{1}{2 t} \sum_{n=0}^{\infty}\left(\begin{array}{c}
1 / 2 \\
n+1
\end{array}\right) \cdot(-4 t)^{n+1} \\
& =\sum_{n=0}^{\infty} \frac{1}{n+1}\left(\begin{array}{c}
2 n \\
n
\end{array}\right) t^{n},
\end{aligned}
$$

and so the Corollary follows. 
2. Computationally, two different cases arise in handling the problem of the title; that is, the technique of the conversion depends on whether the variables $z_{1}, z_{2}, \cdots$, $z_{n}, \cdots$ are given as functional expressions of $n$ or they are specified as numerical values, In what follows, we will treat the first case. Our results will then hold under the condition laid down in the first case. In the second case, the computation is much simpler because the variables $z_{n}$ have been assigned numerical values at the outset.

The coefficients $F_{n}$ in (2) can be computed by applying either formula (3) or (10). We consider the two methods separately.

1. The coefficients $F_{n}$ are computed by using (3). If, for a partition of $n$ :

$$
e_{k}=0 \text { and } e_{k+1}>0
$$

for some $k \leqslant n-1$, then

$$
\left(\begin{array}{c}
e_{k}+e_{k+1}-1 \\
e_{k+1}
\end{array}\right)=\left(\begin{array}{c}
e_{k+1}-1 \\
e_{k+1}
\end{array}\right)=0
$$

Thus, these partitions do not contribute to $F_{n}$ and can be discarded. We call the remaining partitions relevant. These are generated by an algorithm defined by the following flow-chart, where the variable "l" denotes the number of nonzero elements in the partition.

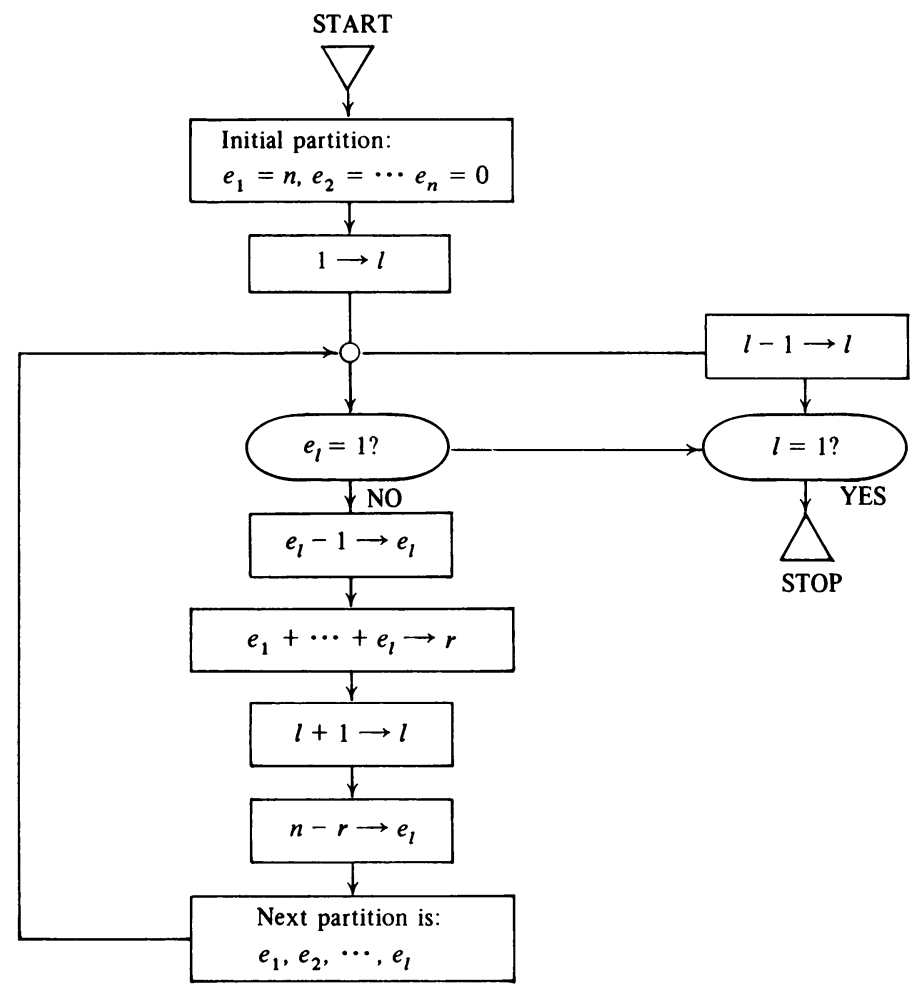


The number of relevant partitions may be denoted by $P_{n}$. Obviously, $P_{n}=1+$ $P_{1}+\cdots+P_{n-1}$ and therefore $P_{n}=2^{n-1}$. For each relevant partition, the expression

$$
z_{1}^{e_{1}} \cdot \prod_{k=2}^{n}\left(\begin{array}{c}
e_{k-1}+e_{k}-1 \\
e_{k}
\end{array}\right) z_{k}^{e_{k}}
$$

is computed and their values are accumulated to obtain $F_{n}$. With $n$ increased by 1 , the number of partitions doubles. The average number of factors in $\left(3^{\prime}\right)$ increases too, so the computation time becomes slightly more than double. This limits the actual computation of the $F_{n}$, while there is no memory problem. Carrying out the computation for some numerical examples, the computation of $F_{1}$ to $F_{18}$ on an IBM 1130 took approximately 80 minutes.

2. Formula (10) can be employed to compute the $F_{n}$ recursively. Each $F_{n}$ is a polynomial in the $z_{k}$ 's and is represented in a segment of a one-dimensional array. The end and beginning of this segment are indicated by pointer addresses. Each $F_{n}$ is a sum of terms having the following form:

$$
C \cdot z_{1}^{e_{1}} \cdot z_{2}^{e_{2}} \cdots z_{n}^{e_{n}}
$$

Such a term is represented by writing the constant $C$ in the first word and the exponents $e_{k}$ into the following words. To save memory space, zero-exponents at the end of a term may be omitted and the end of each term is then indicated by assigning a negative sign to the last exponent. Multiplication of two terms is performed as a multiplication of the coefficients and addition of the exponents. The transition from $F_{k}\left(z_{1}, \cdots, z_{k}\right)$ to $F_{k}\left(z_{2}, \cdots, z_{k+1}\right)$ is carried out by shifting the exponents in all terms by one to the right and introducing a zero after the coefficient $C$. During the multiplication of $F_{k}$ and $F_{n-k}$, the product of two terms has to be checked, if it exists already among the terms of $F_{n+1}$. If this is the case, the coefficient of the product is added to the coefficient of that term; otherwise a new term is introduced.

The difficulty of this method lies in the need of memory space. In order to compute $F_{n+1}$, all the $F_{1}, \cdots, F_{n}$ must be available. The table below shows the memory locations for $F_{1}$ to $F_{9}$.

\begin{tabular}{lc|lc}
\hline$F_{i}$ & Memory locations & $F_{i}$ & Memory locations \\
\hline$F_{1}$ & $1-2$ & $F_{6}$ & $112-255$ \\
$F_{2}$ & $3-7$ & $F_{7}$ & $256-575$ \\
$F_{3}$ & $8-19$ & $F_{8}$ & $576-1279$ \\
$F_{4}$ & $20-47$ & $F_{9}$ & $1280-2815$ \\
$F_{5}$ & $48-111$ & &
\end{tabular}


Department of Mathematics Kenyatta University College Nairobi, Kenya, Africa

Universidade Federal do Rio Grande do Sul

Instituto de Fisica

Rua Prof. Luiz Englert

Porto Alegre, R. G. do Sul, Brazil

1. O. PERRON, Die Lehre von den Kettenbrïchen, Chapter 8, München, 1929; Reprint, Chelsea, New York. 